\title{
FREE GROUPS, SYMMETRIC AND REDUCED PRODUCTS
}

\author{
CARLOS R. BORGES \\ (Received 11 September 1978; revised 12 February 1979) \\ Communicated by J. H. Rubinstein
}

\begin{abstract}
We show that, for any Tychonoff space $X$ with base point $\theta$, the infinite symmetric product $S P^{\infty} X$ of $X$ is a subspace of an abelian group $A(X)$ generated by $X$. (This clarifies the continuity of the multiplication in $S P^{\infty} X$.) Furthermore, $S P^{\infty} X$ is a retract of $A(X)$. Analogous results hold for reduced product spaces, with respect to non-abelian groups.
\end{abstract}

Subject classification (Amer. Math. Soc. (MOS) 1970): primary 22 A 99; secondary 54 B 15.

Keywords and phrases: Infinite symmetric products, reduced product spaces, Graev free groups, embeddings and retracts.

\section{Introduction}

Dold and Thom (1958) introduced infinite symmetric products $S P^{\infty} X$ for all spaces $X$ and defined a multiplication on $S P^{\infty} X$ which, they claim, is generally not continuous (Spanier (1959) has a simpler description of $S P^{\infty} X$, starting on p. 158; see footnote on p. 159). It turns out to be continuous for all Tychonoff spaces $X$.

James (1955) introduced reduced product spaces $X_{\infty}$, which really are the non-abelian version of infinite symmetric products. For Tychonoff spaces $X$, all our results for $S P^{\infty} X$ have analogues for $X_{\infty}$ with respect to non-abelian groups.

For the sake of clarity, we will now describe infinite symmetric products and reduced products in a manner which is more convenient to our work. To avoid extensive repetition, we will assume the terminology of Borges (1977).

Let $X$ be a space with base point $\theta$. Let $S P^{0} X=\{\theta\}$. For $n \geqslant 1$, let $U X^{n}$ be the quotient space of $X^{n}$ which consists of unordered $n$-tuples $\left\langle x_{1}, \ldots, x_{n}\right\rangle$. Then let $S P^{n} X$ be the quotient space of $U X^{n}$ which results from identifying each $\left\langle x_{1}, \ldots, x_{n}\right\rangle$ with the tuple obtained from $\left\langle x_{1}, \ldots, x_{n}\right\rangle$ by removing all $\theta$ 's, except that $\langle\theta, \ldots, \theta\rangle=\langle\theta\rangle$. Let $\lambda_{n}: X^{n} \rightarrow S P^{n} X$ be the resultant quotient map. Finally, 
let $S P^{\infty} X=\Sigma_{n} S P^{n} X$. Define a multiplication on $S P^{\infty} X$ by

$$
\left\langle x_{1}, \ldots, x_{n}\right\rangle_{\circ}\left\langle y_{1}, \ldots, y_{n}\right\rangle=\left\langle x_{1}, \ldots, x_{n}, y_{1}, \ldots, y_{n}\right\rangle .
$$

Clearly $\langle\theta\rangle$ is the unit element of this multiplication.

The description of the reduced product $X_{\infty}$ is exactly the same as that of $S P^{\infty} X$, except that all tuples remain ordered. We let $X_{n} \subset X_{\infty}$ correspond to $S P^{n} X \subset S P^{\infty} X$.

\section{Symmetric products}

For any Tychonoff space $X$ with base point $\theta$, let $\beta X$ be the Stone-C compactification of $X$ with base point $\theta$. We will make extensive use of free abelian topological groups, which were not discussed in Borges (1977). To avoid extensive repetition, we adopt the notation of Borges (1977) for free abelian topological groups, except that we replace $x^{-1}$ by $-x, F_{n}(X)$ by $A_{n}(X),(F(X), \mathscr{G})$ by $(A(X), \mathscr{G})$ and $\left(F(X), \mathscr{G}^{\prime}\right)$ by $\left(A(X), \mathscr{G}^{\prime}\right)$ (see p. 362 of Borges (1977)). We also let $\mathscr{G}_{n}=\mathscr{G} \mid A_{n}(X)$ and $\mathscr{G}_{n}^{\prime}=\mathscr{G}^{\prime} \mid A_{n}(X)$. Recall that $\left(A(X), \mathscr{G}^{\prime}\right)$ is a subspace of $(A(\beta X), \mathscr{G})$.

LEMMA 2.1. Let $X$ be a compact Hausdorff space with base point $\theta$. Then each $S^{n} X$ is a subspace of $\left(A_{n}(X), \mathscr{G}_{n}\right)$.

ProOF. Let us consider the commutative diagram

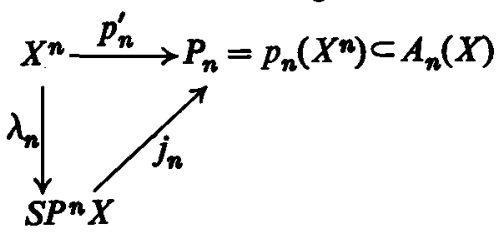

with $p_{n}^{\prime}=p_{n} \mid X^{n}$ and $j_{n}\left(\left\langle x_{1}, \ldots, x_{n}\right\rangle\right)=x_{1}+\ldots+x_{n}$. Since $\lambda_{n}$ and $p_{n}^{\prime}$ (recall that $\left.p_{n}:\left(A_{1}(X)\right)^{n} \rightarrow A_{n}(X)\right)$ are closed continuous maps, we get that $\lambda_{n}$ and $p_{n}^{\prime}$ are quotient maps. Since $j_{n}$ is one-to-one, we then get that $j_{n}$ is a homeomorphism.

THEOREM 2.2. Let $X$ be a Tychonoff space with base point $\theta$. Then each $S P^{n} X$ is a subspace of $\left(A_{n}(X), \mathscr{G}_{n}^{\prime}\right)$.

ProOF. Let us consider the commutative diagrams
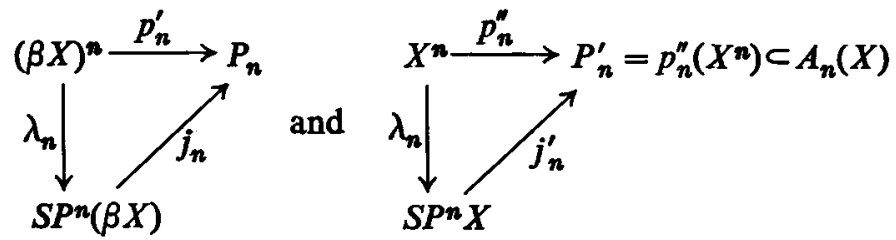
Note that $X^{n}=\left(p_{n}^{\prime}\right)^{-1}\left(P_{n}^{\prime}\right)$ (even though $X^{n} \neq p_{n}^{-1}\left(P_{n}^{\prime}\right)$ ). Since $p_{n}^{\prime}$ is closed and continuous, $P_{n}^{\prime}$ is a subspace of $P_{n}$ and $X^{n}$ is a subspace of $(\beta X)^{n}$, it follows that $p_{n}^{\prime \prime}$ is a quotient map. Of course, the second $\lambda_{n}$ is a quotient map, by definition. Therefore, since $j_{n}^{\prime}$ is one-to-one, we get that $j_{n}^{\prime}$ is a homeomorphism.

LeMma 2.3. Let $X$ be a compact Hausdorff space with base point $\theta$. Then $S P^{\infty} X$ is a subspace of $(A(X), \mathscr{G})$.

Proof. Let us consider the commutative diagram

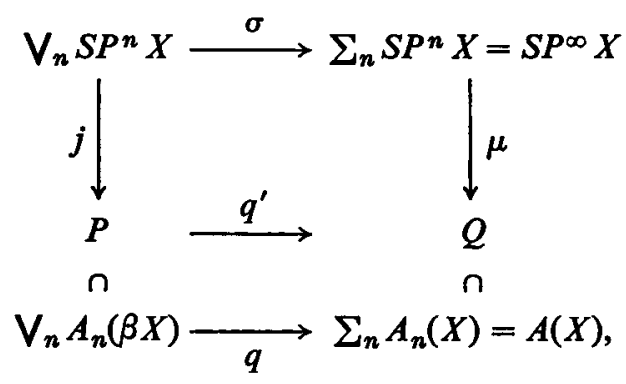

where $j, \mu, \sigma, q^{\prime}$ and $q$ are onto maps, $\sigma$ is the natural quotient map, $j \mid S P^{n} X=j_{n}$ (of Theorem 2.1), $q$ is the natural quotient map (see, for example, Propositon 2.1(a) of Borges (1977)), and $\mu \mid S P^{n} X=j_{n}$.

Note that $Q$ is closed in $A(X)$, because each $Q \cap A_{n}(X)=\mu\left(S P^{n} X\right)$, and $q^{-1}(Q)=P$. Therefore $q^{\prime}$ is also a quotient map. The map $j$ is clearly closed, continuous and one-to-one; therefore $j$ is a homeomorphism. Consequently $\mu$ is a homeomorphism.

THEOREM 2.4. Let $X$ be a Tychonoff space with base point $\theta$. Then $S P^{\infty} X$ is a subspace of $\left(A(X), \mathscr{G}^{\prime}\right)$.

Proor. Let us consider the commutative diagrams

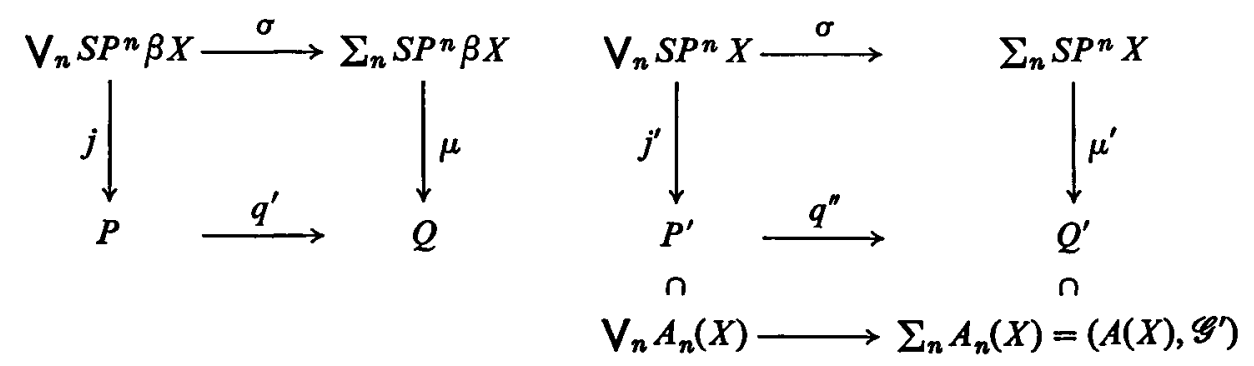


From the proof of Lemma 2.3 we get that, in the first diagram, $q^{\prime}$ is a quotient map. In the second diagram, $\sigma$ is a quotient map (by definition) and $j^{\prime}$ is a homeomorphism because $j^{\prime} \mid S P^{n} X=j_{n}^{\prime}$ (in the proof of Theorem 2.2) is a homeomorphism, for each $n$.

We also have that $Q^{\prime}$ is closed in $\left(A(X), \mathscr{G}^{\prime}\right)$, because $Q^{\prime} \cap A_{n}(X)=\mu\left(S P^{n} X\right)$ and $\mu\left(S P^{n} X\right)=\mu\left(S P^{n} \beta X\right) \cap A(X)$. (Clearly Theorem 2.3 of Borges (1977) remains valid for $\left(A(X), \mathscr{G}^{\prime}\right)$; in particular $\left.\left(A(X), \mathscr{G}^{\prime}\right)=\Sigma_{n} A_{n}(X)\right)$; furthermore, $\left(q^{\prime}\right)^{-1}\left(Q^{\prime}\right)=P^{\prime}$. Therefore $q^{\prime \prime}$ is a quotient map. Consequently $\mu^{\prime}$ is a homeomorphism.

Clearly the embedding of $S P^{\infty} X$ in $\left(A(X), \mathscr{G}^{\prime}\right)$ is such that the multiplication in $S P^{\infty} X$ is the restriction of the multiplication in $\left(A(X), \mathscr{G}^{\prime}\right)$. Therefore, we get the following.

COROLLARY 2.5. If $X$ is a Tychonoff space with base point $\theta$, then the multiplication in $S P^{\infty} X$ is continuous.

We complete this section by showing that, for the class of $k_{\omega}$-spaces, $S P^{\infty} X$ is a retract of $(A(X), \mathscr{G})$ and, for the class of Tychonoff spaces, $S P^{\infty} X$ is a retract of $\left(A(X), \mathscr{G}^{\prime}\right)$.

THEOREM 2.6. Let $X$ be a Hausdorff $k_{\omega}$-space with base point $\theta$. Then $S P^{\infty} X$ is a retract of $(A(X), \mathscr{G})$.

ProOF. For $a \in X$, let $\hat{a}=a$; for $a=-b$, with $b \in X$, let $\hat{a}=b$. Define $r: A(X) \rightarrow S P^{\infty} X$ by $r\left(k_{1} a_{1}+\ldots+k_{j} a_{j}\right)=k_{1} \hat{a}_{1}+\ldots+k_{j} \hat{a}_{j}$.

Clearly $r$ is a retraction. To show that $r$ is continuous, let us consider the following commutative diagram

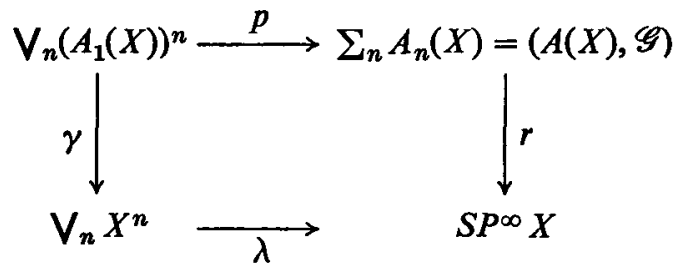

where $\gamma\left(a_{1}, \ldots, a_{n}\right)=\left(\hat{a}_{1}, \ldots, \hat{a}_{n}\right)$ and $\lambda \mid X^{n}=\lambda_{n}$ (in the proof of Lemma 2.1). Clearly $\gamma$ and $\lambda$ are continuous, and $p$ is a quotient map, by Proposition 1.2(a) of Borges (1977). Consequently, $r$ is continuous.

THEOREM 2.7. Let $X$ be a Tychonoff space with base point $\theta$. Then $S P^{\infty} X$ is a retract of $\left(A(X), G^{\prime}\right)$. 
Proof. First note that $S P^{n} X$ is a subspace of $S P^{n} \beta X$. (Note that the map $\lambda_{n}:(\beta X)^{n} \rightarrow S P^{n} \beta X$ is closed and continuous, which implies that

$$
\lambda_{n} \mid X^{n}: X^{n} \rightarrow\left(S P^{n} X \text {, subspace topology from } S P^{n} \beta X\right)
$$

is a quotient map, because $X^{n}=\lambda_{n}^{-1}\left(S P^{n} X\right)$. Since $\lambda_{n}: X^{n} \rightarrow S P^{n} X$ is also a quotient map, by definition, we then get that $S P^{n} X=\left(S P^{n} X\right.$, subspace topology from $S^{n} \beta X$ ).)

For any $Y$, let $r_{n}=r \mid A_{n}(Y)$. From Theorem 2.6 we get that

$$
r_{n}: A_{n}(\beta X) \rightarrow S P^{n} \beta X
$$

is continuous. Consequently, $r_{n}: A_{n}(X) \rightarrow S P^{n} X$ is also continuous. Since $\left(A(X), \mathscr{G}^{\prime}\right)=\Sigma_{n} A_{n}(X)$, we get that $r:\left(A(X), \mathscr{G}^{\prime}\right) \rightarrow S P^{\infty} X$ is continuous.

\section{Reduced products}

It is easy to see that the preceding proofs remain valid for the non-abelian analogues. Therefore, we state the main results for reduced products, without proof or further comment.

THEOREM 3.1. Let $X$ be a Tychonoff space with base point $\theta$. Then $X_{\infty}$ is a subspace of $\left(F(X), \mathscr{G}^{\prime}\right)$.

THEOREM 3.2. Let $X$ be a Tychonoff space with base point $\theta$. Then $X_{\infty}$ is a retract of $\left(F(X), \mathscr{G}^{\prime}\right)$.

\section{References}

C. R. Borges (1977), 'Free topological groups', J. Austral. Math. Soc. Ser. A 23, 360-365.

A. Dold and R. Thom (1958), 'Quasifaserungen and unendliche symmetische Produkte', Ann. of Math. 67, 239-281.

J. P. Hardy, S. A. Morris and H. B. Thompson (1976), 'Applications of the Stone-Cech compactification to free topological groups', Proc. Amer. Math. Soc. 55, 160-164.

I. M. James (1955), 'Reduced product spaces', Ann. of Math. 62, 170-197.

E. Spanier (1959), 'Infinite symmetric products, function spaces, and duality', Ann. of Math. 69, 142-198.

B. V. S. Thomas (1974), 'Free topological groups', General Topology and Appl. 4, 51-72.

Department of Mathematics

University of California

Davis, California 95616

U.S.A. 